\title{
Maritimité et paysage culturel maritime : les apports du modèle " Any-artefact "
}

Maritimity and the maritime cultural landscape. Contribution of the "Anyartefact" model

\section{Sylvain Laubé}

\section{(2) OpenEdition}

Journals

Édition électronique

URL : https://journals.openedition.org/artefact/10228

DOI : $10.4000 /$ artefact. 10228

ISSN : 2606-9245

Éditeur :

Association Artefact. Techniques histoire et sciences humaines, Presses universitaires du Midi

Édition imprimée

Date de publication : 7 octobre 2021

Pagination : 293-316

ISBN : 978-2-8107-0755-3

ISSN : 2273-0753

\section{Référence électronique}

Sylvain Laubé, « Maritimité et paysage culturel maritime : les apports du modèle « Any-artefact » », Artefact [En ligne], 14 | 2021, mis en ligne le 07 octobre 2021, consulté le 07 octobre 2021. URL : http:// journals.openedition.org/artefact/10228; DOI : https://doi.org/10.4000/artefact.10228

\section{(c) (†) $\odot$}

Artefact, Techniques, histoire et sciences humaines est mise à disposition selon les termes de la Licence Creative Commons Attribution - Pas d'Utilisation Commerciale - Pas de Modification 4.0 International. 


\section{Maritimité et paysage culturel maritime : les apports du modèle "Any-artefact»}

\section{Sylvain Laubé}

\section{Résumé}

À partir de l'analyse des différentes définitions données dans le domaine de la géographie et du patrimoine (avec le terme « maritimité ») ou celui de l'archéologie maritime (avec le terme " maritimity »), une proposition plus précise est apportée, articulée avec les concepts de " paysage culturel maritime » et de « lieu de savoir ». Le couplage avec le méta-modèle « Any-artefact », développé à Brest depuis 2012 (dans le cadre de travaux de recherche interdisciplinaires en histoire des techniques et humanités numériques), nous a paru pertinent pour rendre opérante cette définition dans le domaine de l'histoire et du patrimoine des paysages culturels maritimes, dans le sens où « Any-artefact » est dédié à létude comparée des paysages culturels portuaires en considérant que leurs formations et leurs évolutions sont le résultat des activités humaines.

\section{Mots-clés}

modélisation des connaissances, paysages culturels, histoire des sciences et des techniques, humanités numériques

95 Sylvain Laubé, « Maritimité et paysage culturel maritime : les apports du modèle "Any-artefact" », Artefact, 14, 2021, p. 293-316. 


\section{Maritimity and the maritime cultural landscape. Contribution of the "Any-artefact" model}

\section{Abstract}

From the analysis of the different definitions of «maritimity » given and used in the field of geography, heritage or maritime archaeology, a more precise definition is proposed, articulated with the concepts of « maritime cultural landscape » and " lieu de savoir ». The coupling with the meta-model « Any-artefact", developed in Brest since 2012 (in the framework of interdisciplinary research works in history of techniques and digital humanities), seemed to us relevant to make this definition operational in the field of history and heritage of maritime cultural landscapes, in the sense that «Any-artefact » is dedicated to the comparative study of port cultural landscapes by considering that their formation and evolution are resulting of human activities.

\section{Keywords}

knowledge modelling, cultural landscape, history of science and technology, digital humanities

L es questions de recherche qui sont abordées ici se situent dans un contexte de recherche pluridisciplinaire entre laboratoires d'histoire des sciences et techniques et d'informatique, initiée en 2012 dans le cadre du groupe SemanticHPST ${ }^{1}$. Les objectifs étaient alors de proposer des modèles de connaissances (des ontologies pour le web sémantique) dédiés à l'histoire des sciences et des techniques à partir de trois terrains d'études : la correspondance de H. Poincaré ${ }^{2}$, le concept d'énergie et le complexe militaro-industriel du port-arsenal de Brest ${ }^{3}$. À Brest, les travaux

1. Bruneau, Garlatti et al., 2015.

2. Voir par exemple Bruneau, 2014 ; Lasolle, Bruneau et al., 2020.

3. Voir Laubé, 2009a ; Rohou, Laubé et al., 2017 ; Laubé, Garlatti et al., 2017 ; Laubé, Garlatti et al., 2018. 
de recherche se sont élargis, à partir de 2015, en considérant les modèles de connaissances concernant l'histoire de l'activité humaine dans les paysages industriels culturels et leur restitution dans des environnements virtuels ${ }^{4}$. L'interdisciplinarité se situe aussi lors de la " traduction " d'un modèle en histoire en modèle informatique avec la contrainte d'associer à un concept donné une définition univoque afin de permettre l'interopérabilité des données historiques. Cette contrainte conduit de manière assez vertueuse à interroger les concepts sollicités (ici " maritimité ", " paysage culturel maritime ") et utilisés dans les modèles sémantiques et virtuels comme à chercher à les clarifier le cas échéant, en s'appuyant sur les travaux développés antérieurement dans d'autres domaines.

Ainsi, l'archéologue Christer Westerdahl pose-t-il de manière explicite la question en 2007 :

Qu'est-ce qui est maritime? Y a-t-il quelque chose d'exclusivement maritime ? Est-ce que pourrions-nous aboutir à autre chose qu'à un commentaire pragmatique $a d$ hoc ? Eh bien, la réponse à toutes ces questions doit être un non catégorique. [...] La perspective humaine, après tout, se compose toujours de la mer et de la terre, qui fonctionnent en même temps et se définissent mutuellement dans la conscience humaine. Le problème est donc de savoir comment définir ce qui est spécifiquement maritime par rapport à ce qui est spécifiquement terrestre. Cela ne va pas du tout de soi ${ }^{5}$.

Les différentes définitions données dans le domaine de la géographie et du patrimoine (avec le terme "maritimité ») ou celui de l'archéologie maritime (avec le terme " maritimity ") présentant un certain flou, il nous a semblé intéressant d'examiner de plus près ce terme et de chercher à apporter une proposition plus précise articulée avec les concepts de "paysage culturel maritime » et de " lieu de savoir ». Pour la rendre opérante dans le domaine de l'histoire et du patrimoine des paysages culturels maritimes,

4. Voir Querrec, Laubé et al., 2018 ; Abiven, 2019.

5. Westerdahl, 2007, p. 191. Traduit par l'auteur. «What is maritime? Is there anything exclusively maritime? Could we possibly finish up with anything other than a pragmatic ad hoc comment? Well, the answer to all of these questions must be an emphatic no. [...] The human perspective, after all, always consists of both sea and land. They function contemporaneously and so define each other in the human consciousness. The problem is, therefore, how to define the specifically maritime in relation to what is specifically land-oriented or, if you like, terrestrial. This is not at all self-evident. " 
le couplage avec le méta-modèle "Any-artefact ", développé à Brest nous a paru pertinent à présenter dans le sens où il est dédié à l'étude comparée des paysages culturels portuaires en considérant que leurs formations et leurs évolutions sont le résultat des activités humaines. Une première partie sera ainsi consacrée à ces aspects théoriques en cherchant à définir au mieux les concepts avec lesquels nous travaillons. La seconde, plus courte, donnera un exemple simple d'une application du modèle " Anyartefact " qui montre de quelle manière la " maritimité " associée à un artefact (ici, un engin de levage) lorsqu' elle se combine avec d'autres cultures dans le domaine industriel constitue un indicateur d'un changement de paradigme.

\section{Du concept de " maritimité " à celui de « lieu de savoir»}

Le concept de "maritimité » a pour référence première l'ouvrage dirigé par les géographes F. Péron et J. Rieucau à l'issue d'un colloque tenu en 1993 à Brest ${ }^{6}$. De manière explicite, il concerne la «maritimité » contemporaine (" aujourd'hui ») induite notamment par les nouvelles activités liées aux domaines du loisir et du tourisme ainsi que l'installation de nouveaux rapports au patrimoine des sociétés littorales. Dans son introduction, F. Péron indique l'approche retenue :

Une réflexion sur la maritimité invite donc à méditer, non sur les activités humaines directement induites par le milieu maritime (productions marines, transports maritimes, loisirs de bord de mer), au sens où le géographe André Vigarié parle de 'maritimisation' des économies littorales, mais sur la diversité des formes de sensibilité au milieu côtier et maritime et sur l'évolution des façons de percevoir la mer, l'estran, la côte qui conditionnent les activités maritimes des différents groupes sociaux considérés ${ }^{7}$.

Cette " maritimité " constitue un socle théorique souvent cité dans de multiples travaux plutôt situés dans le domaine de la géographie et du patrimoine littoral, bien que ce terme conserve un certain flou ${ }^{8}$ :

\footnotetext{
6. Péron et Rieucau, 1996.

7. Péron et Rieucau, 1996, p. 14.

8. Rubio-Ardanaz, Geistdorfer et al., 2014 ; Chalier, 2019.
} 
La maritimité est un vocable commode pour désigner la variété des façons de s'approprier la mer, en insistant sur celles qui s'inscrivent dans le registre [...] des représentations collectives.[...] Parler de maritimité, c'est inviter à réfléchir sur les constructions sociales et culturelles qui ont été édifiées par les groupes humains pour organiser leurs relations à la mer, pour s'en protéger, pour la socialiser, pour la baliser, pour l'aimer. Ces constructions s'ancrent bien entendu dans la profondeur historique, mais en même temps, elles sont évolutives. S'interroger sur la maritimité contemporaine, c'est réfléchir sur les rapports entre le présent et le passé, dans une perspective de compréhension des reconversions actuelles du maritime, mises en relation avec les aspirations et les besoins profonds de notre société.

À la manière de F. Péron, nous la définissons comme l'ensemble des représentations des littoraux et de la mer dont un groupe social est porteur; ces représentations concernent (i) l'image que cette population se forme de ces milieux, (ii) l'idée qu'elle se fait des ressources qu'ils peuvent offrir ou des plaisirs qu'ils peuvent procurer, et (iii) les savoir-faire et techniques qui permettent de s'y insérer, de les exploiter ou d'en jouir ${ }^{10}$.

En 2010, sans référence aucune aux travaux précédents des géographes, le terme de "maritimity " est convoqué par Tuddenham ${ }^{11}$ dans le champ des paysages culturels maritimes et l'archéologie maritime en réponse à la question posée par C. Westerdahl en $2007^{12}$. Ainsi Tuddenham considère que :

La [ "maritimity "] telle que je la définis et l'utilise est le résultat de l'identification et du tri entre les affaires terrestres et maritimes. La question de savoir ce qui est maritime, comme l'a demandé Westerdahl, est assez complexe, et la maritimité, telle que je l'utilise, ne contribue en aucun cas à une définition plus précise. Mais elle traite de la relation entre la mer et la terre, une question centrale lorsqu'il s'agit d'archéologie maritime et de paysages culturels maritimes. Toutefois, c'est le processus de tri entre la

9. Chalier, 2019 (citant une version traduite en espagnol de Péron et Rieucau, 1996, p. 14).

10. Dantas, 2011.

11. Tuddenham, 2010.

12. Westerdahl, 2007. 
terre et la mer qui est mis en avant, et non le fait de savoir si un phénomène doit être qualifié de maritime ou de terrestre.

La maritimité doit donc être comprise comme une catégorie de compréhension, et non comme quelque chose ayant des qualités empiriques ${ }^{13}$.

Ces définitions, dont les contours restent très larges, semblent ainsi s'inscrire essentiellement dans le champ d'une culture idéelle (la dimension matérielle n'apparaît pas), d'une part, par une procédure mentale de tri (Tuddenham) qui conduit à un traitement de deux pôles (mer et terre) et de leurs relations (et, donc, la construction cognitive et sociale des représentations d'un individu ou d'un groupe social) et, d'autre part, par la variété des "façons de s'approprier la mer ", comme nous l'avons plus haut, et l'ensemble des représentations portées par un groupe social (Péron et Rieucau).

Ces deux définitions (situées dans un monde idéel) sont recouvertes par la définition des savoirs que donne C. Jacob où l'on retrouve l'idée d'agir sur le monde et d'interagir avec lui (s'approprier la mer) avec la différence fondamentale que la multiplicité des formes de savoirs s'inscrivent dans des espaces idéels et aussi matériels :

Je définis les savoirs comme un champ de l'expérience humaine, individuelle et collective, plus précisément comme l'ensemble des procédures mentales, discursives, techniques et sociales par lesquelles une société, les groupes et les individus qui la composent, donnent sens au monde qui les entoure et se donnent les moyens d'agir sur lui ou d'interagir avec lui. Ces procédures concernent aussi bien le monde visible que le monde invisible, le monde matériel que le monde immatériel, le monde naturel et humain, le monde du vivant et de l'inerte, ces différentes dimensions, et les oppositions qui les corrèlent, étant elles-mêmes des constructions

13. Tuddenham, 2010, p. 8. Traduit par l'auteur. "Maritimity as I define and use it is the result of identification and sorting between terrestrial and maritime affairs. The question what is maritime as Westerdahl asked is aquite complex one, and maritimity as I use it doesn't contribute in any sense to help further with a more precise definition. But it does discuss the relationship between sea and land, a central issue when it comes to maritime archaeology and maritime cultural landscapes. Though, it is the sorting process between Land and Sea which is kept in focus, not whether a phenomenon is to be labelled maritime or terrestrial. Maritimity is then to be understood as a category of understanding, not something with empirical qualities. " 
culturelles. [...] Ces procédures de sémantisation du monde, par la pensée, le langage ou l'action, sont fondamentalement sociales. Les savoirs s'incarnent dans des individus ou des communautés qui se voient reconnaître des compétences particulières, ils se matérialisent dans les artefacts qui en sont le résultat et le vecteur, objets, discours, textes écrits, comme dans les gestes efficaces qui reflètent la performativité d'un savoir-faire ${ }^{14}$.

La culture matérielle et immatérielle en jeu nous amène à proposer ici une définition qui rassemble "maritimité " et " maritimity " en s'appuyant sur le cadre proposé par C. Jacob :

- la « maritimité » est un ensemble de savoirs concernant deux pôles matériels anthropisés (terre et mer), leurs interfaces et leurs relations ;

- la construction et l'exercice de cette "maritimité " sont associés à des lieux de savoirs dans une dynamique « du passage d'un lieu à l'autre, d'un lieu mental à un lieu matériel, d'un lieu individuel à un lieu social, de l'atelier à la bibliothèque, du laboratoire à l'usine ${ }^{15}$ ";

- ces lieux de savoirs sont situés spatialement et temporellement. Ils peuvent être portés par un individu, un groupe social, un artefact, des gestes. Ils ne sont pas forcément situés sur les lieux anthropisés concernés.

\section{Du concept de « lieux de savoir » à celui de " paysage culturel maritime "}

De notre point de vue, ce concept de "maritimité ", de par ses aspects cognitifs et ses lieux de savoirs s'articule alors avec celui de " paysage culturel maritime ", proposé par C. Westerdahl en $1992^{16}$ dans le champ de l'archéologie maritime. Dans l'approche « holistique » qui est préconisée ${ }^{17}$, il est défini de la manière suivante :

14. Jacob, 2014, p. 24-25.

15. Jacob, 2014, p. 107.

16. Westerdahl, 1992, p. 5-14.

17. Westerdahl, 1992, p. 6. Du grec holos : qui forme un tout. Conception de la société qui fait que le tout domine les parties, qui ne sont alors que des composantes secondaires, soudées les unes aux autres, interdépendantes, subordonnées à une fin qui leur préexiste. 
ensemble du réseau de routes maritimes, avec les ports, havres et rades le long de la côte, ainsi que les constructions et autres vestiges de l'activité humaine, sous-marins et terrestres, qui y sont associés. Une perspective plus large inclut également la navigation, la construction navale et la pêche, ainsi que leurs arrière-pays respectifs avec les points nodaux des villes côtières et des routes terrestres, les gués, les ferries et les voies navigables intérieures. Les vestiges matériels font déjà l'objet d'une attention particulière de la part de l'archéologie, mais les aspects cognitifs, notamment la carte mentale avec son paysage toponymique (noms de lieux) sont également nécessaires à la compréhension $[\ldots]^{18}$.

Avec pour résumé, l'équation (Équation 1) :

$$
\text { paysage physique }+ \text { paysage cognitif }=\text { paysage culturel }
$$

Pour la période du XVI ${ }^{\mathrm{e}}$ au XIX ${ }^{\mathrm{e}}$ siècle, une liste de 13 éléments ${ }^{19}$ à prendre en compte est proposée pour composer le paysage culturel maritime, dont les ports et les sites de construction navale. Ce concept de " paysage culturel maritime " a été, depuis 1992, longuement repris et discuté au sein d'une double communauté (archéologie et patrimoine maritime) ${ }^{20}$. La synthèse que donne Caporaso, 2017, permet d'en préciser le contour :

- pour qu'un véritable paysage maritime existe, la société qui y vit doit être rythmée/accordée [attuned] d'une manière ou d'une autre par/à la mer, et constituée une préoccupation importante de la société ;

- le paysage incorpore une combinaison de référents, des constructions

18. Westerdahl, 2007, p. 212-213. Traduit par l'auteur. « the whole network of sailingroutes, with ports, havens and harbours along the coast, and its related constructions and otherremains of human activity, underwater as well as terrestrial. A broader perspective also includes shipping, shipbuilding and fishing and their respective hinterlands, with nodal points of coastal towns and land roads, fords, ferries and inland waterways. Material remains are already focused on by archaeology, but cognitive aspects, including the mental map with its toponymous landscape (place names) [...].»

19. Westerdahl, 2007, p. 213. 1) les principales destinations de navigation, 2) les routes maritimes, 3) les sites de balisage, 4) les marques maritimes, 5) les phares, 6) les sites des pilotes, 7) les ports et havres, 8) les lieux de ballasts, 9) les ports de pêches, 10) les chantiers navals et les lieux de constructions navales, 11) les noms de lieux ayant une signification maritime, 12) les sites fondateurs, les sites d'épaves.

20. Pour une revue de la situation, voir par exemple : Caporaso, 2017, p. 1-6 ; Gusick, Dodds et al., 2019 ; Ford, 2011. 
physiques ou des phénomènes capables d'être perçus, et de signifiants, c'està-dire le sens et la reconnaissance/description/interprétation du référent.

- Les référents courants dans les paysages maritimes peuvent inclure des infrastructures côtières ou portuaires intentionnelles ${ }^{21}$ telles que des docks, des quais, des entrepôts, etc., mais aussi des conditions naturelles éventuellement variables (tels que la topographie, le vent, la marée, les courants) et/ou sensorielles (l'odeur de l'air marin...) ;

- les signifiants courants que l'on retrouve dans les paysages identifiés englobent également les mythes, le folklore, l'histoire orale et les phénomènes codifiés tels que tels que le développement de toponymes ou de normes cartographiques.

- Lorsque les composantes du référent et du signifiant perdurent dans le temps et dans l'espace, un discours culturel est transmis et peut évoluer au fil des générations successives ;

- ces motifs [patterning] liés aux paysages peuvent également être transportés dans d'autres lieux que ceux dans lesquels ils ont été créés. En d'autres termes, la formation et la négociation d'un nouveau paysage sont informées par les expériences passées de ses participants dans un précédent ou autre paysage.

De ce croisement des concepts issus de la géographie, de l'archéologie et du patrimoine maritime, deux aspects sont particulièrement à souligner :

- en reprenant l'« équation » initiale de Westerdahl, on peut poser celleci (Équation 2) :

" paysage physique " + " maritimité » + " lieux de savoir " $=$

" paysage culturel maritime»

où " paysage physique » est un environnement composés de deux pôles anthropisés (terre et mer) incluant leurs interfaces situées spatialement et temporellement ;

- dans une approche holistique, un paysage culturel maritime se pense comme un ensemble d'éléments (référents et signifiants) constituant un système complexe (un tout) présentant des motifs communs [patterning] avec d'autres paysages sur une période donnée.

21. Ces référents produits de l'activité intentionnelle des êtres humains seront appelés "artefact " dans le modèle "Any-artefact ". 
Dernier aspect important et essentiel, Westerdahl, 2007, promeut une discussion sur la théorie de la culture maritime en mettant en exergue comme point de départ :

l'utilisation de concepts tels que la culture, l'identité, voire les modes de vie, semble impliquer que les individus ne peuvent être identifiés comme porteurs que d'une seule culture à la fois. Or, ce n'est pas le cas. Les individus comme les groupes peuvent non seulement connaitre plusieurs cultures, mais aussi en faire partie dans un sens très réel. [...] Je pense que c'est un bon début pour une théorie. Quel est donc l'intérêt de définir la culture maritime ou la culture côtière? Quels sont les avantages d'avoir une "théorie " ? N'est-ce pas un peu compliqué ? En avons-nous besoin ? Je le pense moi-même ${ }^{22}$.

\section{L'objectif de cette théorie est clairement identifié :}

[...] déterminer précisément s'il existe un thème commun, et si oui, en quoi il consiste. [...] La perspective est censée donner une vue générale, l'essence, pour ainsi dire. Un problème particulier est que la théorie, ou une partie de celle-ci, devrait être applicable aux temps préhistoriques ou à d'autres périodes ou régions qui ne peuvent être couvertes par les sources historiques conventionnelles. [...] N'oublions pas, ne serait-ce qu'un instant, lorsque nous parlons de culture, que les porteurs de la culture sont toujours des êtres humains ${ }^{23}$.

On constate que les tentatives de définitions du concept de "maritimité » issues des domaines de la géographie et de l'archéologie maritime s'inscrivent

22. Westerdahl, 2007, p. 192-193. Traduit par l'auteur. «The use of concepts like culture, identity, even ways of life seems to imply that individuals can only be identified as carriers of one culture at a time. This is not true. Individuals as well as groups can not only be acquainted with several cultures, they may in fact be part of them in a very real sense. [...] I think this is a good start for a theory. What, then is the point of defining maritime culture or coastal culture? What are the advantages of having a "theory?" Is it not rather complicated? Do we need it? I do think so myself. "

23. Westerdahl, 2007, p. 193. Traduit par l'ateur. « [...] whether there is a common theme, and if so what it consists of. [...] The perspective is supposed to give a generalized view, the essence, so to speak. A particular problem is that the theory, or part of it, should be applicable to prehistoric times or to other periods or areas which cannot be covered by conventional historical sources. [...] Let us not forget even for a moment, when we talk about culture, that the carriers of culture are always human beings. " 
dans la construction de cadres théoriques présentant une étanchéité entre eux. Par contre, Westerdahl, 1992, 2007, et Jacob, 2014, adoptent tous deux des perspectives théoriques plus générales, associées à une généricité des définitions applicables à différents types de situations concrètes d'études. Cela nous a conduits à proposer ici une définition plus complète et précise (nous semble-t-il) du concept de "maritimité » couplée à une définition du " paysage culturel maritime».

Pour devenir opérant, ce cadre théorique général appliqué à des cas d'études concrets (par exemple l'arsenal de Brest) implique de développer des modèles pour examiner de manière fine les situations à partir des sources historiques ou archéologiques, de collecter les données permettant de spécifier les éléments constitutifs d'un paysage culturel (référents et signifiants). L'hypothèse que les savoirs s'incarnent dans les individus ou les groupes sociaux, comme celle qu'ils se matérialisent dans les artefacts et les activités humaines, rejoint celle que nous avions posée dans les travaux de recherche dans les champs croisés de l'histoire des sciences et techniques, l'histoire comparée des paysages culturels maritimes (en prenant comme définition de " paysage culturel » celle de l'UNESCO ${ }^{24}$ ) et de la modélisation des connaissances, qui ont conduit à l'élaboration du modèle "Any-artefact ${ }^{25}$ ». Une autre hypothèse théorique avait aussi été de considérer :

- dans un premier temps, les ports, les arsenaux en tant que macro-systèmes technologiques complexes ${ }^{26}$ (on retrouve ici une approche holistique) pour élargir ensuite le domaine d'application aux paysages industriels culturels sensoriels ${ }^{27}$;

- qu'une histoire comparée des ports, des arsenaux et par extension des environnements industriels implique qu'il existe des descripteurs communs valides pour ces différents paysages (les référents/signifiants cités plus haut).

24. Mitchell, Rössler et al., 2011.

25. Ce modèle a été élaboré en histoire des sciences et des techniques indépendamment du concept de " maritimité » qui, au-delà de son cadre un peu flou, ne semblait pas assez opérant pour atteindre les objectifs qui étaient de décrire l'évolution des paysages culturels maritimes.

26. En référence aux travaux sur les "Large Technical Systems » : Mayntz et Hughes, 1988.

27. Astic, Gasnier et al., 2020. 
Enfin, l'approche systémique qui a été construite prend en compte les multiples échelles spatiales et temporelles sur la longue durée avec les hypothèses suivantes :

- l'activité humaine (dans l'interaction avec une topographie naturelle initiale) est centrale dans la formation et l'évolution d'un paysage culturel industriel ou maritime, elle est donc située spatialement et temporellement ;

- l'activité humaine implique un environnement matériel et sensoriel (dont des artefacts), des acteurs (groupes ou individus) et des savoirs (couplés à des lieux de savoirs.

"Any-artefact » a été élaboré pour aider à répondre aux questions suivantes du point de vue de l'histoire des sciences et des techniques : i) comment décrire et comprendre les dynamiques d'évolution spatio-temporelle d'un macro-système technologique ? ii) comment décrire une activité humaine? iii) comment relier les échelles d'une activité humaine aux échelles beaucoup plus larges de la dynamique d'évolution du paysage culturel dans laquelle elle se situe?

\section{Modéliser pour interpréter, comprendre le paysage culturel maritime et le restituer : le méta-modèle « Any- artefact »}

Ce modèle en histoire a servi de cadre théorique pour une histoire comparée des ports et des arsenaux dans le cadre de deux thèses, co-encadrées au Centre F. Viète et au LabSTICC, associées à des travaux de modélisation des connaissances (web sémantique et réalité virtuelle) ${ }^{28}$.

"Any-artefact » a été dérivé de l'approche du géographe J. Bird et de son modèle "Anyport " ${ }^{29}$, dédié à l'étude comparée de l'évolution spatiale et temporelle des ports sur la longue durée en six phases en prenant notamment comme référents le développement des quais et des docks ${ }^{30}$. De même qu' "Anyport », "Any-artefact » est un méta-modèle dans le sens où il se

28. Rohou, 2018 ; Abiven, 2019.

29. Bird, 1963.

30. Hoyle, 1968. 
présente comme générique et implique d'être ensuite spécifié (instancié) pour chaque cas d'études. De la même manière, il se veut heuristique et destiné à fournir un outil conceptuel, pour répondre aux problématiques en histoire citées ci-dessus, et des modèles de connaissances (des ontologies en informatique) pour la production de bibliothèques numériques basées sur le web sémantique et la création d'environnement virtuel intelligent $(\mathrm{EVI})^{31}$.

Il présente deux composantes : un modèle d'activité humaine et un modèle de « biographie » d'artefact :

\section{Composante 1 : le modèle d'activité humaine (Fig. 1)}

L'activité humaine est considérée comme une suite d'événements situés spatialement et temporellement qui implique trois concepts (ou référents) organisés en système (artefact, acteur et savoir) que nous définissons ainsi :

- Artefact. "C'est la chose susceptible d'un usage, élaborée pour s'inscrire dans des activités finalisées. [...] L'artefact concrétise une solution à un problème ou à une classe de problèmes socialement posés. ${ }^{32}$ ". Un système d'artefacts agencé de manière intentionnelle est un artefact. Un port, un arsenal (et par extension un " paysage culturel maritime) est considéré un macro-système technologique ${ }^{33}$ et comme un artefact (composé lui-même d'artefacts organisés en système).

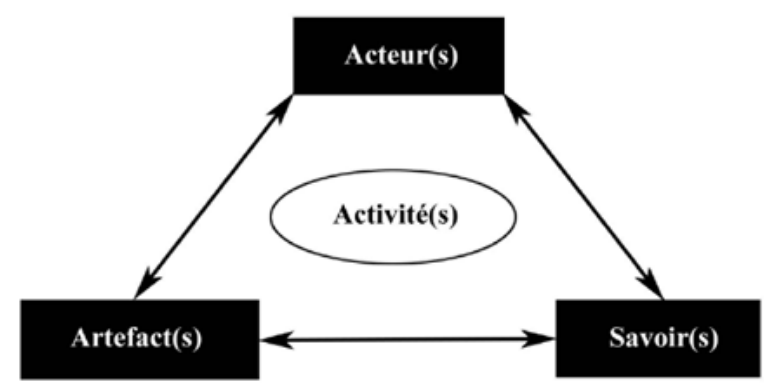

Fig. 1. - Le méta-modèle "Any-artefact »

L'activité humaine implique trois types de référents : artefacts, savoirs et acteurs Conception Sylvain Laubé

31. Abiven, 2019, p. 267-305 ; Querrec, Laubé et al., 2018.

32. Nous reprenons ici la définition de Rabardel, 1995, p. 49.

33. Mayntz et Hughes, 1988. 
- Acteur. Deux types sont distingués : les individus (humains ou animaux) et les entités sociales qui sont des organisations plus ou moins formalisées : des groupes sociaux (un état, une entreprise, un laboratoire, un groupe d'ami.es, etc.) et des "personnages " qui représentent des positions qui sont incarnées par des individus.

- Savoirs. Il s'agit des savoirs tels que définis plus haut par C. Jacob en indiquant deux types : les savoirs déclaratifs qui concernent les savoirs énoncés sous diverses formes langagières et les savoirs incarnés (ou incorporés) qu'on ne peut analyser ou décomposer jusqu'au bout, seulement transmissibles par apprentissage ${ }^{34}$. Les savoirs déclaratifs sont à nouveau décomposables en plusieurs types : savoirs factuels, procéduraux, théoriques ${ }^{35}$.

\section{Composante 2 : Le modèle de « biographie » ou " percursus" d'un artefact (Tableau 1)}

Si le terme « biographie » d'objets a fait l'objet de nombreuses publications et discussions sur son intérêt et sa nature épistémologique ${ }^{36}$, il lui sera ici cependant préféré ici le terme "percursus " en notant que "biographie » entretient une analogie avec des concepts du vivant que nous souhaitons éviter $^{37}$. Le vocable "parcours " est parfois aussi sollicité mais se traduit par une grande multiplicité sémantique. Pour ces raisons, nous utiliserons, pour sa neutralité, le mot latin " percursus » dont le mot " parcours » est issu étymologiquement ${ }^{38}$.

Tableau 1. Les étapes du « percursus » d’un artefact

\begin{tabular}{|l|l|}
\hline Etape 1 & Emergence d'un besoin au sein d'un groupe social \\
\hline Etape 2 & $\begin{array}{l}\text { Traduction de ce besoin en problèmes technologiques, } \\
\text { émergence de différentes solutions aux problèmes posés, } \\
\text { choix d'une solution }\end{array}$ \\
\hline Etape 3 & Réalisation de l'artefact \\
\hline Etape 4 & $\begin{array}{l}\text { Usage de l'artefact comprenant les phases d'entretien et } \\
\text { de réparation }\end{array}$ \\
\hline Etape 5 & Arrêt des usages/obsolescence/disparition \\
\hline
\end{tabular}

34. Chamoux, 1978.

35. Krathwohl, 2002.

36. Voir par exemple Bensaude-Vincent, 2012 ; Daston, 2000 ; Bonnot, 2015.

37. Un article est en préparation pour discuter et justifier l'emploi d'un terme neutre vis-à-vis du vivant.

38. https://fr.wiktionary.org/wiki/parcours\#\%C3\%89tymologie 
Chaque étape se traduit par une série d'activités humaines décrites par le modèle de la Figure 1 avec un jeu multiple d'acteurs, de savoirs et d'artefacts. L'émergence de nouveaux besoins (par exemple lors de l'étape 4) peuvent conduire à une boucle itérative qui aura pour effet de déclencher un nouveau cycle pour modifier l'artefact.

Le paysage culturel maritime étant considéré comme un macro-système complexe, "Any-artefact " permet de périodiser le port à partir d'indicateurs (référents) considérés comme pertinents. Dans le cas des ports ou des arsenaux, plusieurs aspects apparaissent comme essentiels : i) les ouvrages maritimes (darse, digue, môle, quai, etc.), ii) les lieux de mobilités (grue, pont, etc.), iii) les lieux de stockage (silos élévateurs, etc.) ${ }^{39}$, iv) les lieux de productions industriels (forges, corderies, etc.) ${ }^{40}$.

\section{La question de la " maritimité " dans un exemple de « paysage culturel maritime " : l'arsenal de Brest}

\section{Un artefact comme lieu de " maritimité » : l'engin de levage}

Chaque étape du " percursus » d'un artefact se traduit par une série d'activités humaines où sont également engagées des acteurs et des savoirs. Dans le cadre d'une comparaison d'artefacts répondant aux mêmes fonctions (ici les engins de levage), ces étapes permettent de situer en quoi ils sont ou non des lieux de "maritimité », de montrer des ruptures (ou des évolutions lentes) notamment par l'implication d'autres lieux de savoirs, non spécifique du paysage culturel maritime. L'exemple des engins de levage montre ainsi un changement industriel à partir de 1870 .

La Figure 2 montre les implantations des engins de levage de charges de fort tonnage (de $10 \mathrm{t}$ à $160 \mathrm{t}$ ) du XVII ${ }^{\mathrm{e}}$ au début du XIX ${ }^{\mathrm{e}}$ siècle ainsi que le type et leurs dates de construction et disparition. Elles révèlent une trace dans un ordre chronologique (1 à 4) de la dynamique de l'évolution du paysage industriel le long du fleuve avec un premier mouvement entrant

39. Voir Rohou, 2018, sur la périodisation des ports de Brest, Mar del Plata et Rosario.

40. Voir Abiven, 2019, sur la question de la modélisation de l'activité et la comparaison des arsenaux de Brest et Venise. 
à l'intérieur de la Penfeld de la fin du XviI ${ }^{\mathrm{e}}$ (première machine à mâter) jusqu'aux années 1860 (grue "Gervaize ") puis un mouvement inverse à partir des années 1880 (grue hydraulique) qui se développera en 1910 (grue électrique) pour aboutir au paysage actuel avec la disparition complète des engins de levages de fort tonnage le long de la Penfeld au profit du front de mer.

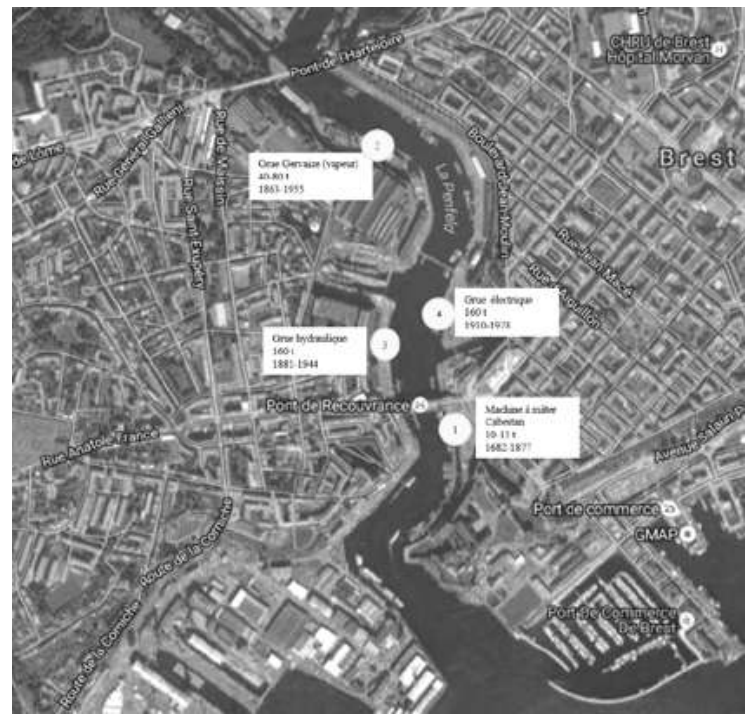

Fig. 2. - Les engins de levage comme indicateurs de l'évolution de l'arsenal

Conception Sylvain Laubé

La machine à mâter (1) et la grue dite "Gervaize » (2) présentent des " precursus " similaires dans le sens où chacune des cinq étapes sont inscrites dans une "maritimité » caractéristiques de la culture industrielle maritime propre à l'arsenal. En effet, l'émergence des besoins (étape 1), la solution technologique (étape 2) comme sa réalisation (étape 3) sont assurées localement en sollicitant des savoirs internes à l'arsenal. Pour la machine à mâter (avec une première version en 1681, puis une seconde améliorée en 1763), il s'agit des techniques classiques d'assemblage de bigues associées à des systèmes de palans qu'on retrouve régulièrement dans le paysage de l'arsenal brestois et des autres arsenaux (Fig. 3). La machine est dédiée aux procédures de mâtage/démâtage des bâtiments à voile de la Marine, l'énergie utilisée étant celle de plusieurs centaines de bagnards sur trois cabestans (étape 4). Son démontage en 1877 (étape 5) est le signal de la fin de la construction navale à voile à Brest. 


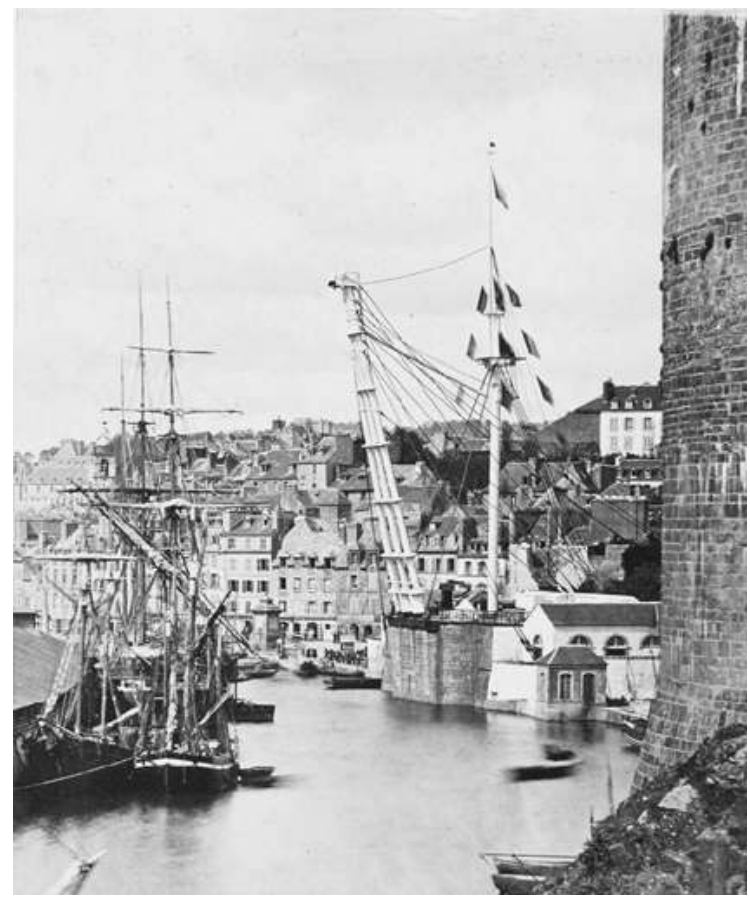

Fig. 3. - La machine à mâter de Brest (1856)

G. Eastman Museum, Geneviève Elisabeth Disderi

https://commons.wikimedia.org/wiki/

Category:Brest_(France)_in_the_1850s

La grue "Gervaize » (Fig. 4) installée en 1863 porte le nom de l'ingénieur qui l'a conçue. Sa notice biographique ${ }^{41}$ indique une activité professionnelle insérée complétement dans la culture industrielle de la construction navale pour la Marine. Sa formation (1835-1839) passe d'abord par Polytechnique puis le corps du Génie Maritime. Il occupe ensuite le poste d'ingénieur du Génie Maritime, notamment chargé de la construction navale et de chaudières (1839-1865) et dirige à partir de 1849 l'atelier des machines à Brest jusqu'en 1861. Il sera ensuite en poste comme Directeur des Constructions Navales (1865-1873) puis Inspecteur du Génie Maritime (1873-1881). Une grue à vapeur (40-80t) sera construite d'après les plans et indications de Gervaize en un seul exemplaire et installée en 1863. Elle constitue un exemple de transfert direct de technologies issues 
de la construction navale au sein de l'arsenal de Brest. Sa fonction sera d'effectuer l'interface entre les grands ateliers des Capucins construits dans les années 1840 et les bâtiments de la Marine, notamment pour le dépôt des chaudières ou des tourelles d'artillerie.

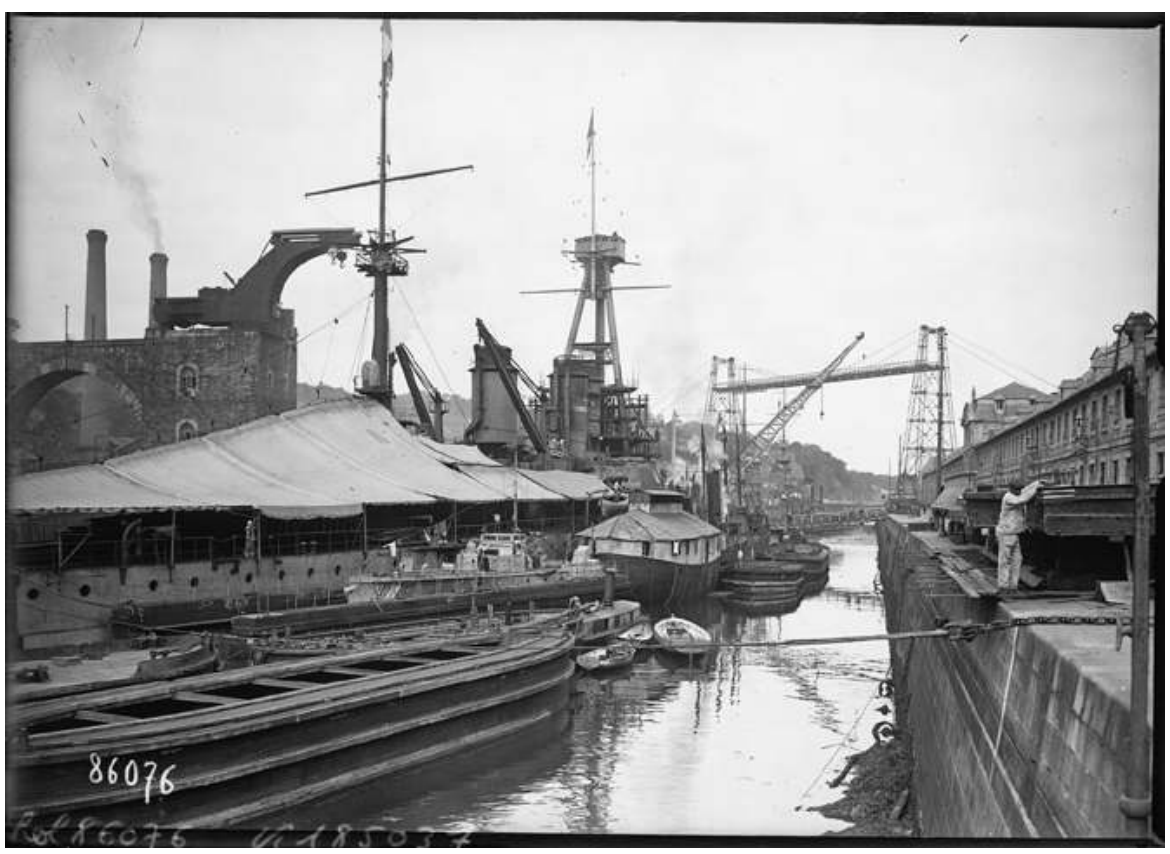

Fig. 4. - La grue « Gervaize » (à gauche). Vue de l'arsenal de Brest en 1923

Photographie de presse, Agence Rol, Agence photographique (commanditaire). Gallica / BnF : https://gallica.bnf.fr/ark:/12148/btv1b53118607z

L'obsolescence de la machine à mâter comme l'insuffisance de la grue "Gervaize » dès la fin des années 1870 traduisent un changement drastique de paradigme en termes de "maritimité " au travers d'un nouveau besoin suivi de l'achat, de l'installation d'une grue hydraulique de 160 t (3) conçue et fabriquée par les constructeurs Bon et Lustremant dans le cadre d'une double commande par la Marine pour les arsenaux de Brest et Toulon ${ }^{42}$. De même en 1910, une grue électrique de 160 t (4) est construite et installée par l'entreprise Fives-Lille. 


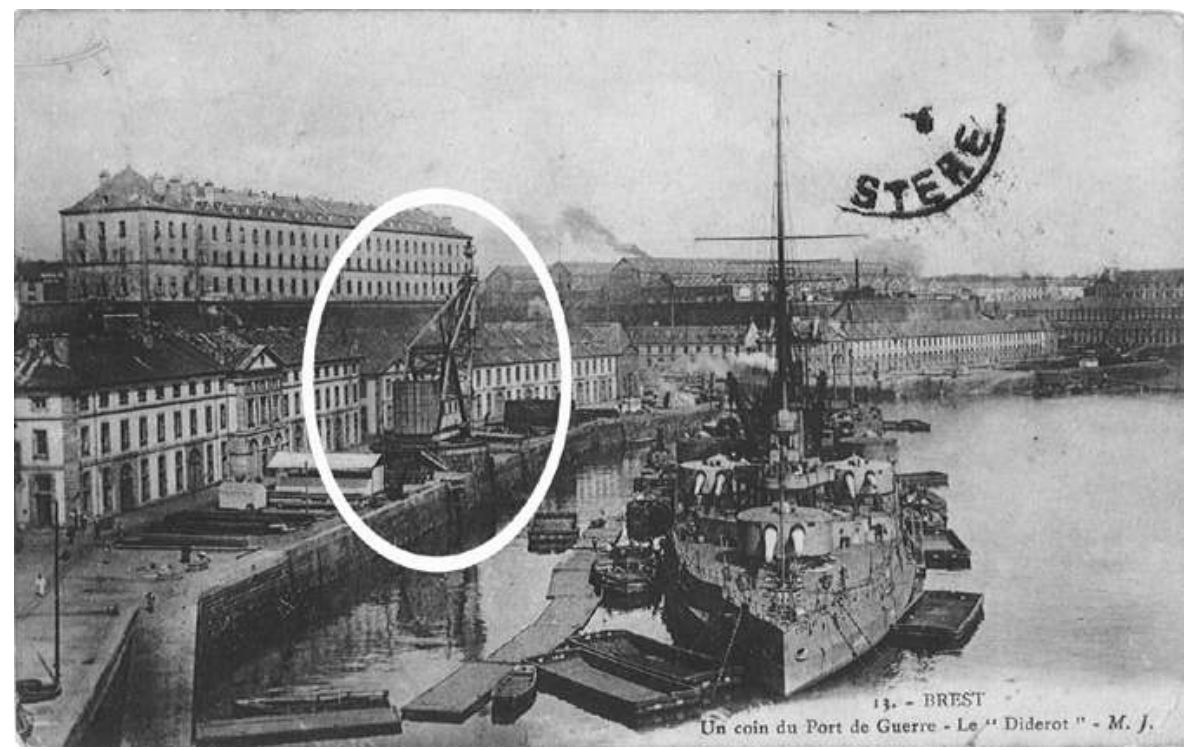

\section{Fig. 5. - La grue hydraulique}

Carte postale « Un coin du Port de Guerre : le Diderot », sans date, Bibliothèque municipale de Brest.

\section{Gallica / BnF : https://gallica.bnf.fr/ark:/12148/btv1b10105638r}

Pour ces deux derniers engins de levage (Fig. 5 et 6), la " maritimité » dans le paysage de l'arsenal s'inscrira à partir de la deuxième moitié du XIX siècle uniquement dans les activités humaines concernant l'émergence et la caractérisation des besoins (étape 1), le choix final de la solution technique (étape 2) et les usages (étape 4). Les solutions techniques (étape 2) et la réalisation (étape 3) étant dévolues à des entreprises dont la " maritimité " n'est pas la culture principale. En effet, la solution technologique, la fabrication et l'installation seront " externalisés " vers des entreprises spécialistes de la construction métallique (Bon et Lustremant, Fives-Lille) et donc en s'appuyant sur des acteurs et savoirs hors de la culture maritime même si leurs produits industriels sont conçus pour répondre à un cahier des charges de la construction navale. Les disparitions (étape 5) avec le démontage de la machine à mâter en 1877 , la destruction en septembre 1944 de la grue hydraulique par fait de guerre, le démontage en 1956 de la grue Gervaize qui n'était déjà plus en service avant-guerre et en 1978 de la grue électrique, traduisent le lent processus d'arrêt de l'activité industrielle 
le long de la Penfeld et dans les grands ateliers construits au XIX ${ }^{\mathrm{e}}$ siècle au profit du front de mer (Laninon) dans une dynamique qui apparaît déjà à la fin du siècle (avec un achèvement en 2004).

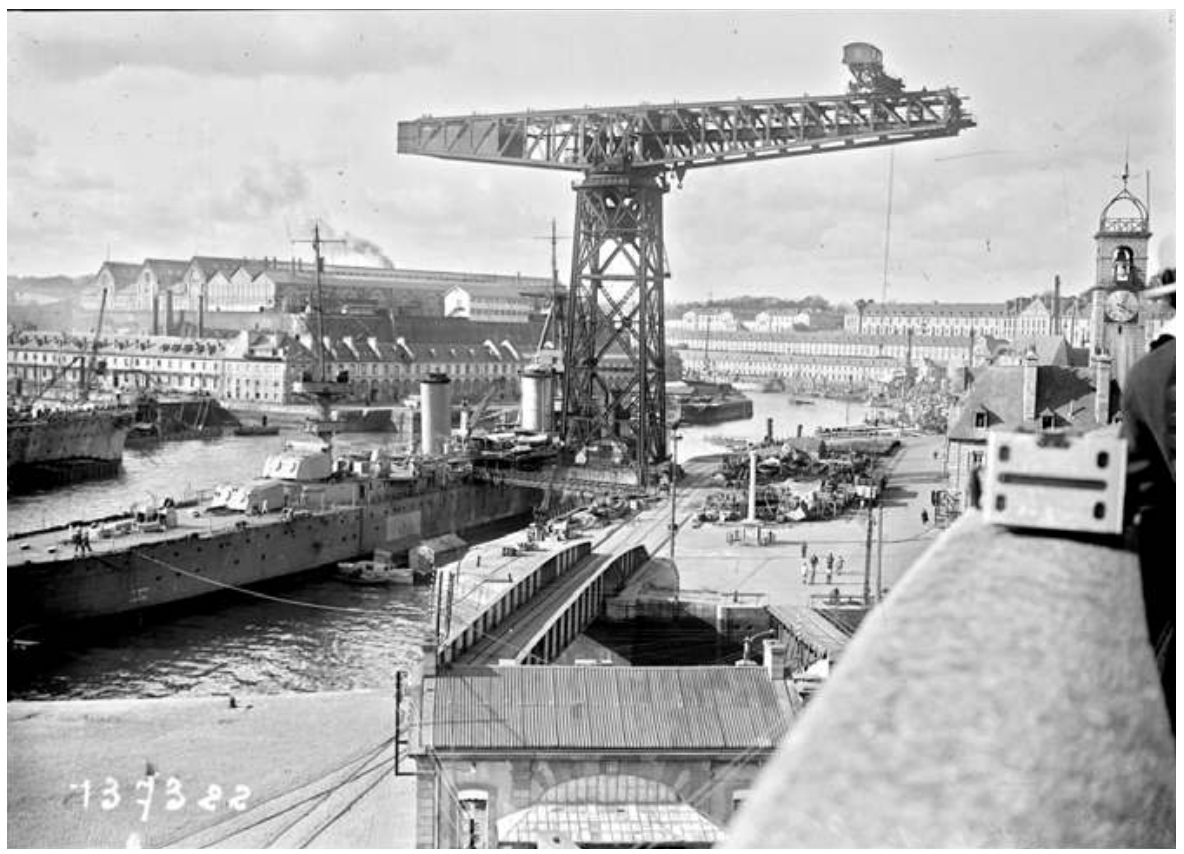

\section{Fig. 6. - La grue électrique}

Vue de l'arsenal de Brest en 1929, Photographie, Agence Rol, Agence photographique (commanditaire).

Gallica / BnF : https://gallica.bnf.fr/ark:/12148/btv1b53214093h

Les " percursus » des engins de levage sont donc des indicateurs à la fois de l'évolution spatio-temporelle de l'arsenal mais aussi des changements de paradigmes industriels. L'étude des "percursus » d'autres artefacts comme les formes de construction, les corderies, les forges, etc. permettent de compléter la dynamique complexe des changements du paysage culturel industriel maritime sur la longue durée, chaque type d'artefacts pouvant se traduire par des changements de paradigmes différents. 


\section{Conclusion et perspectives de recherche}

Le court premier exemple développé à partir du cas des engins de levage montre qu'on peut identifier une réduction de la "maritimité " d'une classe d'artefacts lors de l'externalisation de certaines activités (étape 2 et étape 3) du " percursus ». Ce phénomène n'est pas nouveau et a été mis en évidence par exemple pour la forge des ancres à la fin du XvII ${ }^{\mathrm{e}}$ siècle. Cette externalisation s'est ainsi traduite par une très forte méfiance des officiers de marine et a induit l'émergence d'un nouveau besoin, celui de tester les ancres pour s'assurer de leur qualitét ${ }^{3}$. Les procédures de résolution de ce problème se traduiront par un croisement des cultures et des savoirs maritimes, industriels et savants.

Nous avons discuté un cadre théorique qui associe "maritimité ", "paysage culturel maritime " " paysage physique " et " lieux de savoirs " et chercher à montrer que le modèle "Any-artefact " s'articule en bonne cohérence avec ce cadre pour analyser de manière fine différentes situations historiques. Il s'agit d'un premier résultat exploratoire pour aider à décrire et comprendre les différents régimes de maritimité d'un paysage culturel industriel sur la longue durée. Les travaux engagés dans le cadre du projet ANR Lab In Virtuo ${ }^{44}$ (2021-2024) permettront d'intégrer ce cadre "théorie + modèle " (et d'en discuter la robustesse) pour l'étude des paysages culturels maritimes sensoriels et leur restitution dans des environnements virtuels intelligents réalistes et sensoriels (EVIRS).

\section{Bibliographie}

AвIVen Marie-Morgane, Humanités Numériques et méthodes de conservation et de valorisation du patrimoine maritime. L'exemple des arsenaux de Brest et Venise, Thèse, Université de Bretagne occidentale - Brest, 2019.

Astic Isabelle, Gasnier Marina, et al., « Paysages industriels culturels sensoriels (PICS) et Environnements Virtuels. Vers une nouvelle approche de l'histoire et de l'archéologie industrielle ", [Rapport] 2020, halshs-02613279v2.

Bensaude-Vincent Bernadette, «Vies d'objets», Critique, 2012, n 6, p. 588-598.

BIRD James Harold, The major seaports of the United Kingdom, Hutchinson, London, 1963.

43. Orain et Laubé, 2017.

44. https://scanr.enseignementsup-recherche.gouv.fr/project/ANR-20-CE38-0016 
Bonnot Thierry, "La biographie d'objets: Une proposition de synthèse ", Culture \& Musées. Muséologie et recherches sur la culture, 2015, n² 25, p. 165-183.

Bruneau Olivier, "Web sémantique et HPST : la correspondance d'Henri Poincaré ", Congrès de la Société d'Histoire des Sciences et des Techniques, 2014.

Bruneau Olivier, Gaillard Emmanuelle, Lasolle Nicolas, et al., "A SPARQL query transformation rule language-application to retrieval and adaptation in case-based reasoning ", International Conference on Case-Based Reasoning, Springer, Cham, 2017, p. 76-91.

Bruneau Olivier, Garlatti Serge, Guedj Muriel, et al., «SemanticHPST: applying semantic web principles and technologies to the history and philosophy of science and technology ", dans European Semantic Web Conference, Springer, Cham, 2015, p. 416-427, hal-01214698.

Caporaso Alicia (dir.), Formation Processes of Maritime Archaeological Landscapes, Springer, Cham, 2017.

Chalier Gustavo, "Algunas consideraciones acerca de Puerto Belgrano como área patrimonial: concientización y revalorización para el fortalecimiento del sentido de pertenencia ", Res Gesta, 2019, n 55, p. 10-29.

Chamoux Marie Noëlle, « La transmission des savoir-faire: un objet pour l'ethnologie des techniques?", Techniques et culture, 1978, n 3, p. 46-83.

Dantas Eustogio, "La maritimité chez les indiens du Brésil », Géographie et cultures, ${ }^{\circ}$ 78, 2011, p. 75-96.

Daston Lorraine (dir.), Biographies of Scientific Objects, The University of Chicago Press, Chicago and London, 2000.

Ford Ben (dir.), The archaeology of maritime landscapes, Springer Science \& Business Media, New-York Dordrecht Heidelberg London, 2011.

Gusick Amy E., Dodds Tricia, Jaffke Denise, et al., " Defining Maritime Cultural Landscapes ", California Archaeology, 2019, vol. 11, n² 2, p. 139-164.

Hoyle Brian S., "East African seaports: An application of the concept of 'anyport' ", Transactions of the Institute of British Geographers, 1968, p. 163-183.

ЈАсов Christian, Qu'est-ce qu'un lieu de savoir ?, OpenEdition Press, Marseille, 2014.

KRAThwohl David R., "A revision of Bloom's taxonomy: An overview ", Theory into practice, 2002, t. 41, n 4, p. 212-218.

Lasolle Nicolas, Bruneau Olivier, Lieber Jean, et al., "Assisting the RDF Annotation of a Digital Humanities Corpus using Case-Based Reasoning ", International Semantic Web Conference, Springer, Cham, 2020, p. 617-633. 
LaubÉ Sylvain, « Modélisation des documents numériques pour l'histoire des techniques : une perspective de recherche ", Documents pour l'histoire des techniques. Nouvelle série, 2009a, $\mathrm{n}^{\circ} 18$, p. 37-41.

Laubé Sylvain, Garlatti Serge et Querrec Ronan, " Histoire des paysages culturels industriels et humanités numériques: le modèle d'activité humaine ANY-ARTEFACT ", "Capitalismo en el desierto: Materialidades, población y territorios en Atacama (ss. XIX-XXI)", III Jornada de Antropología e Historia, 2017.

Laubé Sylvain, Garlatti Serge, Querrec Ronan et al., «ANY-ARTEFACT-O : an ontology developed for history of industrial cultural landscape ", 2nd Data for History workshop, Pôle histoire numérique (Digital history department) of the LARHRA laboratory, May 2018, Lyon, hal-01950647.

LaubÉ Sylvain, Victor Charles Eudore Gervaize (1817-1882). Notice biographique, [Contrat] Brest Métropole Océane, 2009b, halshs-03192154.

Mayntz Renate, Hughes Thomas P., The development of large technical systems, Max Planck Institute for the Study of Societies, Köln, 1988.

Mitchell Nora, Rössler Mechtild et Tricaud Pierre-Marie, Paysages culturels du patrimoine mondial: guide pratique de conservation et de gestion, Unesco, Paris, 2011.

Orain Arnaud, LaubÉ Sylvain, «Scholars versus Practitioners?: Anchor Proof Testing and the Birth of a Mixed Culture in Eighteenth-Century France ", Technology and culture, 2017, vol. 58, $\mathrm{n}^{\circ}$ 1, p. 1-34.

PÉRon Françoise et Rieucau Jean (éd.). La maritimité aujourd'hui, L'Harmattan, Paris, 1996.

Querrec Ronan, Laubé Sylvain et al., "Lab in Virtuo : un Environnement Virtuel Intelligent pour l'histoire et le patrimoine des paysages culturels industriels ", dans S. Eusèbe, T. Nicolas, V. Gouranton, R. Gaugne (dir.), Archéologie : imagerie numérique et $3 D$ : actes du 3e séminaire scientifique et technique de l'Inrap, 26-27 juin 2018, Rennes, 2018. Disponible en ligne sur https://sstinrap.hypotheses.org/487.

Rabardel Pierre, Les hommes et les technologies : approche cognitive des instruments contemporains, Armand Colin, Paris, 1995.

Rohou Bruno, Laubé Sylvain, GarLatti Serge, "The port history ontology ", European Conference on Advances in Databases and Information Systems, Springer, Cham, 2017, p. 363-372.

Rohou Bruno, Modélisation des ports de Brest (France), Rosario et Mar del Plata (Argentine) en tant que macro systèmes technologiques complexes : application à la modélisation des connaissances pour l'histoire des sciences et des techniques, Thèse Université de Bretagne occidentale - Brest, 2018, tel-02106927v2. 
Rubio-Ardanaz Juan Antonio, Geistdorfer Aliette, Matxin Jon Ruigomez, Antropologia y maritimidad: Entramados y constructos patrimoniales en el Abra y Ría de Bilbao, Museo Marítimo Ría de Bilbao, Bilbao, 2014.

Tuddenham David Berg, « Maritime cultural landscapes, maritimity and quasi objects ", Journal of Maritime Archaeology, 2010, vol. 5, n 1, p. 5-16.

Westerdahl Christer, "The maritime cultural landscape ", International Journal of Nautical Archaeology, 1992, vol. 21, n 1, p. 5-14.

Westerdhal Christer, "Fish and ships: towards a theory of maritime culture", Deutsches Schiffahrtsarchiv, vol. 30, 2007, p. 191-236.

\section{L'auteur}

Sylvain Laubé est maître de conférences au Centre François Viète/UBO et au Centre Européen de Réalité Virtuelle. Il développe des recherches sur l'histoire comparée des paysages culturels industriels, maritimes sensoriels et des humanités numériques. Il co-dirige plusieurs thèses sur le sujet. Il anime avec Ronan Querrec (CERV) le projet ANR Lab In Virtuo (2021-2024). Il a publié avec Arnaud Orain, "Scholars versus practitioners?: Anchor proof testing and the birth of a mixed culture in eighteenth-century France", Technology and culture, 2017, vol. 58, $n^{\circ}$ 1, p. 1-34 et avec Bruno Rohou et Serge Garlatti, "The port history ontology", European Conference on Advances in Databases and Information Systems, Springer, Cham, 2017, p. 363-372.Courriel : sylvain.laube@univ-brest.fr 